\title{
Piezo-ICSI for Human Oocytes
}

\author{
Kenichiro Hiraoka ${ }^{1,2,3}$, Manami Isuge ${ }^{1}$, Yuko Kamada ${ }^{1}$, Tetsuya Kaji ${ }^{1}$, Takako Suhara ${ }^{1}$, Akira Kuga ${ }^{2}$, Kumi Ohuchi ${ }^{1}$, Masaru \\ Hayashi $^{1}$, Kiyotaka Kawai ${ }^{1,2,3}$ \\ ${ }^{1}$ Kameda Medical Center ${ }^{2}$ Kameda IVF Clinic Makuhari ${ }^{3}$ Tokyo Medical and Dental University
}

\section{Corresponding Author}

Kenichiro Hiraoka

ken.msn.hiraoka@gmail.com

\section{Citation}

Hiraoka, K., Isuge, M., Kamada, Y., Kaji, T., Suhara, T., Kuga, A., Ohuchi, K., Hayashi, M., Kawai, K. Piezo-ICSI for Human Oocytes. J. Vis. Exp. (170), e60224, doi:10.3791/60224 (2021).

\section{Date Published}

April 20, 2021

DOI

$10.3791 / 60224$

URL

jove.com/video/60224

\section{Abstract}

Since the first successful pregnancies achieved by intracytoplasmic sperm injection (ICSI) were reported, ICSI has become an essential technique in assisted reproductive technology (ART). ICSI uses micropipettes with a spiking tip to penetrate the zona pellucida and membrane. Then, the cytoplasm is usually aspirated into the micropipette for membrane breakage (conventional-ICSI). The survival and fertilization rates of mouse oocytes after conventional-ICSI were as low as $16 \%$ and $8 \%$, respectively. Kimura and Yanagimachi applied a piezo drive unit, mercury, and a micropipette with a flat tip for mouse ICSI. The membrane breakage could be performed semi-automatically by combining these types of equipment without cytoplasmic aspiration into the micropipette (piezo-ICSI). These authors reported significantly higher survival and fertilization rates $(80 \%$ and $78 \%)$ compared to those of conventional-ICSI (16\% and $8 \%)$. Therefore, the piezo-ICSI may be effective not only for mouse oocytes but also for human oocyte ICSI. However, only five papers are available that assessed the effectiveness of piezo-ICSI compared to conventional-ICSI for human oocytes. All of these five papers reported significantly higher fertilization rates compared to those of conventional-ICSI. The goal of the piezo-ICSI protocol described here is to improve the clinical results of ICSI compared to the conventionalICSI.

\section{Introduction}

Since Dr. Palermo reported the first successful pregnancies achieved by intracytoplasmic sperm injection (ICSI) ${ }^{1}$, ICSI has become an essential technique in assisted reproductive technology (ART). ICSI uses micropipettes with a spiking tip to penetrate the zona pellucida and membrane. Then, the cytoplasm is usually aspirated into the micropipette for membrane breakage (conventional-ICSI). The survival and fertilization rates of mouse oocytes after conventionalICSI were as low as $16 \%$ and $8 \%$, respectively ${ }^{2}$. Kimura and Yanagimachi applied a piezo drive unit, mercury, and a micropipette with a flat tip for mouse $|C S|^{2}$. The membrane breakage could be performed semi-automatically by combining these types of equipment (piezo-ICSI). These authors reported significantly higher survival and fertilization 
rates $(80 \%$ and $78 \%)$ of piezo-ICSI compared to those of conventional-ICSI $(16 \% \text { and } 8 \%)^{2}$. One of the reasons for the better results obtained in the piezo-ICSI may be the process of membrane breakage. The piezo-ICSI can stably and semi-automatically break the membrane without cytoplasmic aspiration into the micropipette. These results suggested that the cytoplasmic aspiration into the micropipette during membrane breakage is invasive for mouse oocytes. Therefore, the piezo-ICSI may be effective not only for mouse oocytes but also for human oocyte ICSI.

However, only five available papers have assessed the effectiveness of piezo-ICSI as compared to conventionalICSI for human oocytes. All five of these papers reported significantly higher fertilization rates with piezoICSI compared to fertilization rates with conventional$|C S|^{3,4,5,6,7}$ (Table 1). Therefore, the piezo-ICSI may improve the survival and fertilization rates compared to conventional-ICSI. The goal of the piezo-ICSI protocol described here is to improve the clinical results of ICSI compared to conventional-ICSI.

\section{Protocol}

The protocol of piezo-ICSI described below follows the guidelines of the human research ethics committee at Kameda Medical Center.

\section{Equipment and preparation}

1. Use a piezo-ICSI system composed of a drive unit, an operation box, a footswitch, and a controller. Use any type of inverted microscope or three-axis micromanipulator. Attach the piezo drive unit to the micropipette holder, and place the footswitch on the floor.
Set the INTENSITY level to 2 and the SPEED level to 1 on the operation box.

2. Use a commercially available piezo-ICSI micropipette with a flat tip. Fill a micropipette with the operation liquid (fluorocarbon-based fluid) to a length of $20 \mathrm{~mm}$. Avoid mixing any air bubbles in the operation liquid. Aspirate any air bubbles or excess operation liquid towards the back of the pipette with the liquid filling device.

3. Install the micropipette into the injection micropipette holder. If the injection micropipette holder has a silicon tube, insert the head of the pipette about $5 \mathrm{~mm}$ into the silicon tube. Tighten the holder cap and firmly fix the micropipette.

4. Attach the drive unit to the manipulator and fix firmly to avoid any rotation caused by its weight.

5. Position the micropipette in the microscope field. Operate the injector and push the operation liquid to the head of the micropipette so that any air inside the head of the pipette is pressed out. Confirm that there is no air left inside the head of the pipette with the naked eye rather than with a microscope.

6. Prepare the ICSI dish on the glass-bottom dish as shown in Figure 1. Prepare two microdroplets of $7 \%$ polyvinylpyrrolidone solution (PVP): the smaller microdroplet is for the preparation of micropipette $(5 \mu \mathrm{L})$ and the other one is for sperm selection $(10 \mu \mathrm{L})$. Prepare another droplet made in the center of the glass-bottom dish from the buffered medium; this microdroplet is for the oocyte and is used for sperm injection $(8 \mu \mathrm{L})$.

7. Insert the head of the micropipette into a 7\% PVP droplet and coat the inner wall of the micropipette. Operate the injector vigorously, moving it along the interfacial boundary between the operation liquid and the PVP. 
Rinse the inside of the micropipette with PVP about 800 $\mu \mathrm{m}$ from the head of the pipette.

1. Repeat this procedure ( 3 or 4 times) until the interfacial boundary slides smoothly.

\section{Sperm immobilization}

1. Set the INTENSITY level to 2 and the SPEED level to 1 on the operation box. Apply the piezo pulse from the drive unit attached to the micropipette holder when the footswitch is turned on. When applying the piezo pulse during the procedure, the "Piezo pulse" icon appears on the upper right of the video.

2. Collect the motile sperm by using a density gradient and hold the collected motile sperm into the buffered medium. Aspirate the buffered medium holding the collected motile sperm about 2-3 $\mu \mathrm{L}$ by using a pipette. Then inject this medium into the lower side of the 7\% PVP microdroplet. Select the sperm under 400x magnification.

1. Attach the micropipette tip to the tail of the selected sperm and then drive the piezo drive unit. Typically, repeat the driving 3 times for one sperm immobilization. After the sperm immobilization, aspirate the sperm into the micropipette tail first.

\section{Piezo-ICSI operation (an oocyte with a high- stretching ability membrane)}

1. Keep the sperm's head at a position about one full length sperm back from the head of the micropipette. While driving the piezo drive unit (about 5 times per one oocyte), advance the micropipette to carry out the puncture without deformation of the zona pellucida. When the micropipette tip reaches the inner layer of the zona pellucida and then the hollowed-out piece of the zona pellucida is completely aspirated into the micropipette, puncture of the zona pellucida is completed.

2. Remove the hollowed-out piece of the zona pellucida inside the micropipette and at the same time move the sperm to the head of the micropipette. Push the micropipette in and stretch the cytoplasmic membrane until about $90 \%$ of the oocyte diameter.

3. When it is sufficiently stretched, start up the piezo drive one time to rupture the membrane. Membrane breakage is completed when it is confirmed that the membrane has rebounded.

4. After membrane breakage, inject the sperm's head into the oocyte. After the sperm's head is surely injected into the cytoplasm, pay attention not to add unnecessary liquid.

\section{Piezo-ICSI operation (an oocyte with a low- stretching ability membrane)}

NOTE: We explain how to deal with an oocyte with a lowstretching ability membrane which may spontaneously get ruptured during the stretching.

1. Perform the same action as the oocyte with a highstretching ability membrane up to the point of stretching the cytoplasmic membrane.

2. If the membrane is spontaneously ruptured during the action, inject the sperm's head from the position of the micropipette without pushing it further forward to avoid the degeneration of oocytes after sperm injection.

3. After the sperm's head is surely injected into the cytoplasm, pay attention not to add unnecessary liquid. 


\section{Representative Results}

The conventional-ICSI and piezo-ICSI described above were performed on 10,488 mature oocytes (1,744 cycles) by 4 embryologists. Of these, 3,522 oocytes (587 cycles) were inseminated by conventional-ICSI between January 2013 and September 2016 and 6,966 oocytes (1,157 cycles) were inseminated by piezo-ICSI between October 2016 and December 2020 at Kameda Medical Center. Table 1 shows the average age of the women, the fertilization rates, the survival rates, the degeneration rates, the day- 5 blastocyst rates, and the average number of cryopreserved blastocysts per cycles of conventional-ICSI and piezo-ICSI. Although the average women's age of piezo-ICSI was higher as compared to that of conventional-ICSI, the rates of fertilization, survival, and blastocyst and the average number of cryopreserved blastocysts per cycles of piezo-ICSI were significantly higher as compared to those of conventional-ICSI (Table 2).

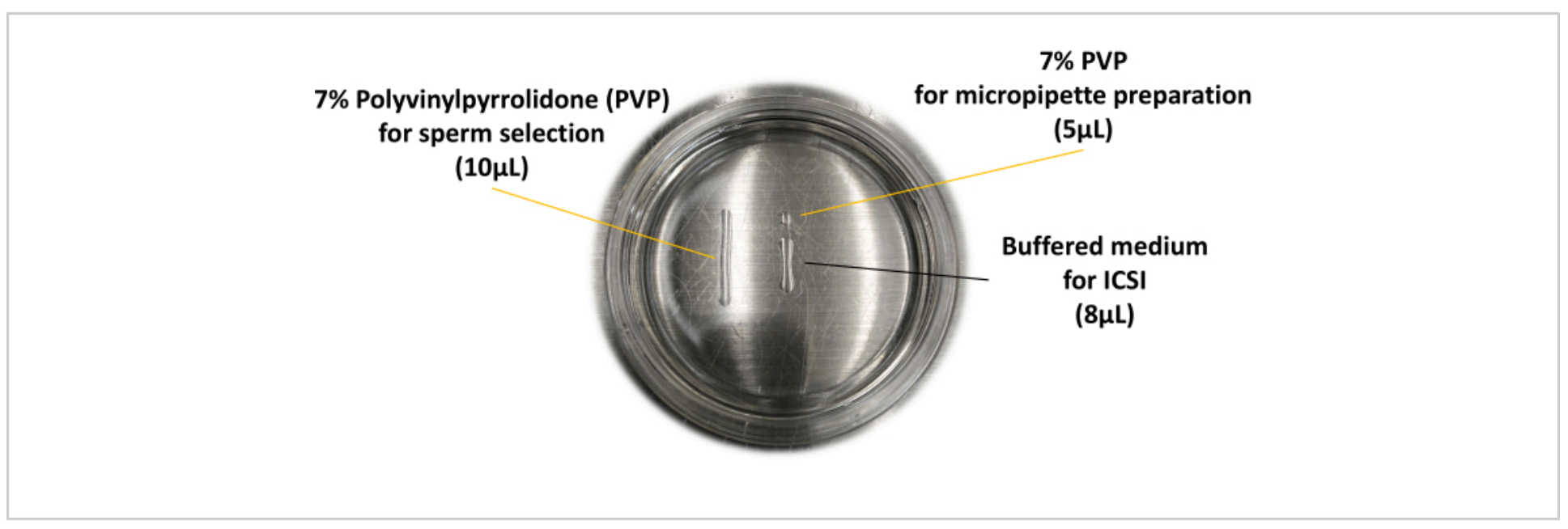

Figure 1: ICSI dish used for piezo-ICSI. The layout of the microdroplets is described here. Please click here to view a larger version of this figure. 


\begin{tabular}{|c|c|c|}
\hline Study & conventional-ICSI & piezo-ICSI \\
\hline Yanagida et al. (1999) & $66 \%^{\mathrm{a}}$ & $79 \%^{\mathrm{b}}$ \\
\hline Takeuchi et al. (2001) & $83 \%^{\mathrm{a}} \%^{\mathrm{b}}$ & $89 \%^{\mathrm{b}}$ \\
\hline Hiraoka et al. (2015) & $68 \%^{\mathrm{a}}$ & $75 \%^{\mathrm{b}}$ \\
\hline Furuhashi et al. (2019) & $64 \%^{\mathrm{a}}$ & $84 \%^{\mathrm{b}}$ \\
\hline Fujii et al. (2020) & $71 \%^{\mathrm{a}}$ & \\
\hline $\mathrm{ab}<0.05$ in the same line & & \\
\hline
\end{tabular}

Table 1: Comparison of fertilization rates between conventional-ICSI and piezo-ICSI. In all reports, significantly higher fertilization rates were observed in the piezo-ICSI as compared to those of the conventional-ICSI.

\begin{tabular}{|c|c|c|c|}
\hline & conventional-ICSI & piezo-ICSI & P \\
\hline Average age of women & $38.6 \pm 0.2$ & $39.2 \pm 0.2$ & - \\
\hline No. of cycles & 587 & 6,966 & $<0.01$ \\
\hline No. of oocytes & 3,522 & $5,411(77.7)$ & $<0.01$ \\
\hline No. of fertilization (\%) & $2,352(66.8)$ & $6,767(97.1)$ & $<0.01$ \\
\hline No. of survival (\%) & $3,303(93.8)$ & $199(2.9)$ & $<0.01$ \\
\hline No. of degeneration (\%) & $219(6.2)$ & $2,787(51.5)$ & $<0.01$ \\
\hline No. of blastocyst (\%) & $915(38.9)$ & $5.1 \pm 0.2$ & \\
\hline $\begin{array}{c}\text { Average no. of cryopreserved } \\
\text { blastocysts per cycles }\end{array}$ & $3.8 \pm 0.2$ & & \\
\hline
\end{tabular}

Table 2: Clinical results of Kameda Medical Center comparing the efficacy of conventional-ICSI and piezo-ICSI. In all comparison items, significantly better results were observed in the piezo-ICSI as compared to those of the conventional-ICSI.

\section{Discussion}

The results demonstrated that the fertilization rates, the survival rates, the day- 5 blastocyst rates, and the average number of cryopreserved blastocysts of the piezo-ICSI were significantly higher compared to those of conventional-ICSI.
There are 2 critical steps in the piezo-ICSI protocol. The first step is to firmly twist the holder cap of the pipette holder. The second step is to maintain the aspirated PVP volume into the micropipette less than 300 micrometers. Loosely twisting the holder cap of the micropipette holder and aspiration 
of PVP into the micropipette more than 300 micrometers (one full length of sperm is about 60 micrometers) cause difficulties in the sperm immobilization, zona opening, and membrane breakage because of the decreasing piezo power. In particular, we always have to pay attention to the aspirated PVP volume into the micropipette during the procedure. This is the limitation of the piezo-ICSI.

It is essential to place a small amount of heavy liquid as an operation liquid inside the micropipette to create the piezo power. In the previous reports, mercury was used as an operation liquid $2,3,4$. However, a fluorocarbonbased fluid can be used instead of mercury for safe used instead $^{5,6,7,8,9,11}$. The fluorocarbon-based liquid has a specific gravity of 1.8 , and is colorless, transparent, and nonwater soluble. The operation liquid has direct contact with the PVP holding the sperm. The exposure of the PVP to the operation liquid may have some negative effects on the sperm or oocyte. However, the rates of fertilization, survival, day-5 blastocyst of piezo-ICSI were significantly higher as compared to those of conventional-ICSI in the present study. We believe the risk from the direct contact between the operation liquid and the PVP is quite low.

We previously measured the time of the micropipette preparation with an oil injector and an air injector. The average times for micropipette preparation of the oil injector and the air injector were 233 and 106 seconds, respectively $(P<0.05)^{8}$. The micropipette preparation time with the air injector is lower because it is oil-free. The presence of the oil extends the micropipette preparation time because of its stickiness. If air bubbles get mixed in the oil area inside the micropipette after inserting the micropipette into the pipette holder, the pipette must be discarded because the piezoICSI will not work. We also counted the number of wasted micropipettes during micropipette preparation. The average number of wasted micropipettes per patient for the oil injector and air injector was $0.28 \pm 0.56$ and $0 \pm 0(P<0.05)$, respectively ${ }^{8}$. However, there was no significant difference between the oil and air injectors in the rates of survival $(99 \%$ and $99 \%$ ), fertilization ( $89 \%$ and $90 \%$ ), and good quality day-3 embryos (61\% and 61\%) after piezo-ICSI ${ }^{8}$. We wasted an extra 3.2 hours and 25 micropipettes in the oil injector group (90 patients) compared to the air injector group (90 patients) during the study ${ }^{8}$. The results indicate that using an air injector instead of an oil injector is a good modification of piezo-ICSI.

The piezo-ICSI can break the membrane semi-automatically without cytoplasmic aspiration into the micropipette. This membrane breakage procedure is stable and easy. The piezo-ICSI could also contribute to shortening the ICSI training period. We previously assessed the effect of the piezo-ICSI on the rates of survival, fertilization, blastocyst for a young embryologist. The young embryologist received piezo-ICSI practice 30 times before starting the clinical ICSI treatment. We compared the survival, fertilization, blastocyst rates of young and senior embryologists for their 1st 100 oocytes. The survival, fertilization, blastocyst rates of the young and senior embryologists were $100 \%$ and $97 \%, 87 \%$ and $91 \%, 47 \%$, and $57 \%$, respectively ${ }^{9}$. There was no significant difference between the young and the senior embryologists in all comparison items. These results indicated that the piezo-ICSI has significance in the membrane breakage procedure that can contribute to shortening the ICSI training period of a young embryologist.

Some investigators reported the clinical efficiency of intracytoplasmic morphologically selected sperm injection (IMSI). Setti et al. compared the clinical outcomes between 
ICSI and IMSI in a meta-analysis study ${ }^{10}$. There was no significant difference in the fertilization rates between the ICSI and IMSI groups ${ }^{10}$. However, the top-quality embryo rate of the IMSI group was significantly higher compared to that of the ICSI group ${ }^{10}$. To the best of our knowledge, no study has examined the impact of IMSI combined with piezo-ICSI on fertilization and embryo development. We retrospectively investigated the effects of sperm selection magnification (400x vs. $1,200 x)$ on fertilization and embryo development in piezo-ICSI. The fertilization rate of the $1,200 x$ group (92\%) was significantly higher as compared to that of the 400x group $(77 \%)(P=0.0002)^{11}$. The good quality day-3 embryo rate of the $1,200 x$ group (64\%) was significantly higher as compared to that of the $400 x$ group $(46 \%)(P=0.0021)^{11}$. We tried to select the sperm without vacuoles in the sperm's head under 1,200x magnification. This procedure might select the sperm with a lower degree of sperm DNA fragmentation ${ }^{12}$. Therefore, the IMSI may be a new application of piezo-ICSI.

Here, we described a protocol for piezo-ICSI that can improve ICSI outcomes without missing viable oocytes in human ART.

\section{Disclosures}

The authors have nothing to disclose.

\section{References}

1. Palermo, G., Joris, H., Devroey, P., Van Steirteghem, A.C. Pregnancies after intracytoplasmic injection of single spermatozoon into an oocyte. Lancet. 340 (8810), 17-18 (1992).

2. Kimura, Y., Yanagimachi, R. Intracytoplasmic sperm injection in the mouse. Biology and Reproduction. 52 (4), 709-720 (1995).
3. Yanagida, K. et al. The usefulness of a piezomicromanipulator in intracytoplasmic sperm injection in humans. Human Reproduction. 14 (2), 448-453 (1999).

4. Takeuchi, S. et al. Comparison of piezo-assisted micromanipulation with conventional micromanipulation for intracytoplasmic sperm injection into human oocytes. Gynecologic and Obstetric Investigation. 52 (3), 158-162 (2001).

5. Hiraoka, K., Kitamura, S. Clinical efficiency of Piezo-ICSI using micropipettes with a wall thickness of $0.625 \mu \mathrm{m}$. Journal of Assisted Reproduction and Genetics. 32 (12), 1827-1833 (2015).

6. Furuhashi, K. et al. Piezo-assisted ICSI improves fertilization and blastocyst development rates compared with conventional ICSI in women aged more than 35 years. Reproductive Medicine and Biology. 18 (4), 357-361 (2019).

7. Fujii, Y., Endo, Y., Mitsuhata, S., Hayashi, M., Motoyama, H. Evaluation of the effect of piezo-intracytoplasmic sperm injection on the laboratory, clinical, and neonatal outcomes. Reproductive Medicine and Biology. 19 (2), 198-205 (2020).

8. Hiraoka, K. et al. Clinical efficiency and safety of piezoICSI with pneumatic injector. Fertility and Sterility. 100 (3), S91 (2013).

9. Ibayashi, M. et al. The impact of Piezo-ICSI on clinical results for trainee embryologist new to the ICSI [Japanese]. Journal of Clinical Embryologist. 21 (1), 1-8 (2018).

10. Setti, A.S. et al. Intracytoplasmic sperm injection outcome versus intracytoplasmic morphologically selected sperm injection outcome: a meta-analysis. 
Reproductive BioMedicine Online. 21 (4), 450-455 (2010).

11. Hiraoka, K., Otsuka, Y., Ishikawa, T., Kawai, K., Harada, T. Effect the sperm selection magnification $(400 \mathrm{x}$ vs $1,200 x)$ on fertilization results and embryo development in human Piezo-ICSI. Fertility and Sterility. 108 (3), e147 (2017).

12. Franco, J.G. Jr. et al. Significance of large nuclear vacuoles in human spermatozoa: implications for ICSI. Reproductive BioMedicine Online. 17 (1), 42-45 (2008). 